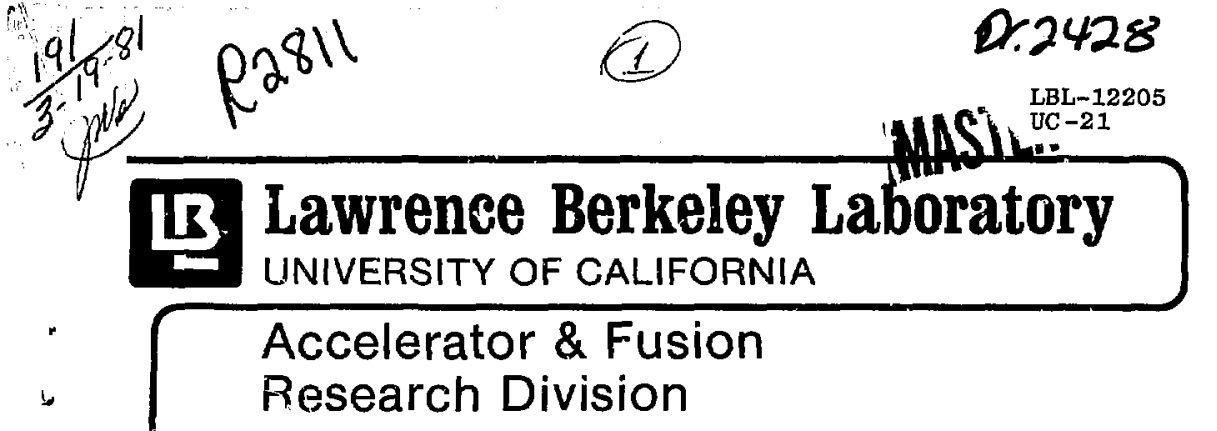

A PARTICLE SIMULATION CODE FOR LONGITUDINAL DYNAMICS OF HEAVY ION BEAMS

A. Sterulieb,'L. Smith, L.J. Laslett, J. Bisognano, and I. Haber

January 1981

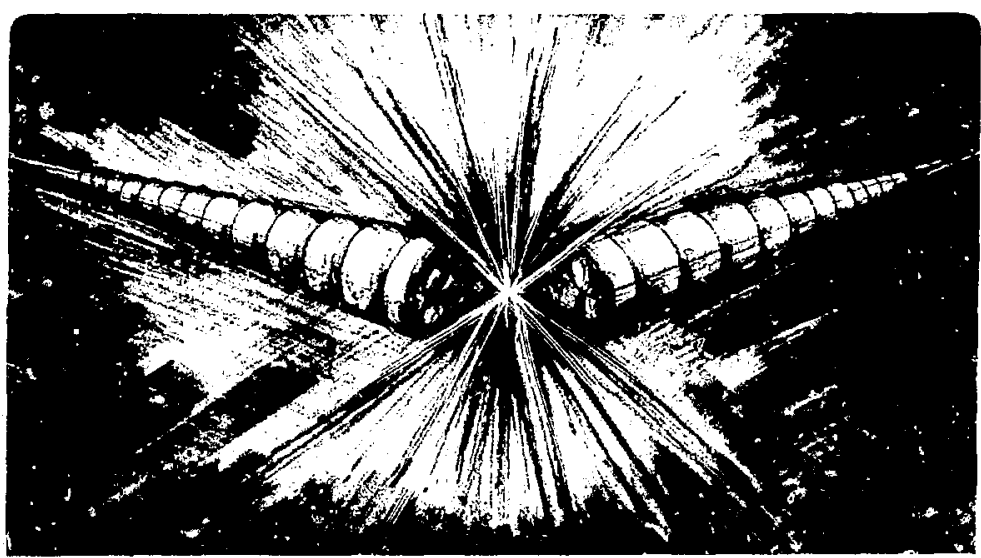

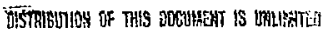


LBL-12205

HI-FAN-144

\title{
A PARTICLE SIMULATION CODE FOR LONGITUDINAL DYNAMICS OF HEAVY ION BEAMS*
}

\section{A. Sternlieb, L. Smith, L.J. Laslett, J. Bisognano Accelerator and Fusion Research Division Lawrence Berke ley Laboratory University of California Berkeley, California 94720}

\author{
I. Haber \\ Naval Research Laboratory \\ Washington, 0.C. 20375
}

January 198I

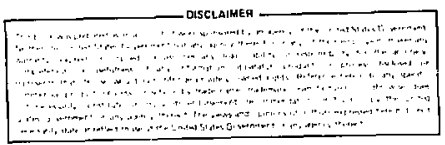

* This work was supported by the Director, Office af Energy Research, Office of Inertial Fusion, Research Division of the U. S. Department of Energy under Contract No. W-7405-ENG-48. 


\section{A Particle Simulation Code for Longitudinal}

Dynamics of Heavy Ion Beams

A. Sternlieb, L. Smith, L.J. Laslett, J. Bisognano, I. Haber**

\section{Abstract}

A particle computer simulation code is presented, designed to describe the longitudinal motion of charged particles in a nonneutralized beam. The code is 1-d, electrostatic, and is capable of simulating coasting or bunched beams, cold or warm, with arbitrary wall impedance. External focusing forces are introduced in the bunched beam case. A variety of distribution functions can be represented. The code is self-consistent and it can follow both the linear and nonlinear evolution of a beam.

**Naval Research Laboratory, Washington, D.C. 20375 
In the last few years, heavy ion beams have been suggested as possible drivers in an inertial conf inement fusion scheme. (1) Because of the relatively high nonneutralized currents involved, space-charge effects are important during the acceleration and transport of the beam through the highly evacuated focusing system. The transverse stabitity of such beams has been investigated to a certain extent, using both theoretical anaiys is ${ }^{(2)}$ and computer simulation. (3) The agreement found is encouraging.

Recently, an attempt has been made to formulate analytically the problem of longitudinal stability of a bunched beam. Certain stationary distribution functions $f\left(x, v_{x}\right)$ have been proposed ${ }^{(4)}$ and 1 inearly analyzed for stability against perturbations. (4b) A linear analys is for a cold bunch and arbitrary density distribution has also been perfomed. ${ }^{(5)}$ In the above stability analyses, the transverse and longitudinal motions of the particles in the bunch are assumed to be completely decoupled. In addition, a resistive wall term was introduced in the motion equations. (5) The general conclusion until now is that, with a resistive wall as a perturbation a bunched heam is linearly stable.

However, it is clear that the analytical approach to the longitudinal stability problem has serious shortcomings, especially in using various disputable approximations and assumptions. Therefore, the need arises for a simulation program, which can study in a self-consistent way both the linear and nonlinear longitudinal stability and evolution of a bunched beam. In the following we describe a particle code which has been designed to study those phenomena not amenable to theoretical analysis.

\section{The Simulation code}

\section{(a) General Remarks}

The simulation code is one-dinensional, electrostatic, and it follows the motion of several thousands of particles in the self-consistent electric field generated by space-charge (plus possible externally imposed forces). The simulation system is chosen in a reference frame moving with the beam. The time evolution is performed according to a first-order time-centered leap-frog scheme, as follows: 


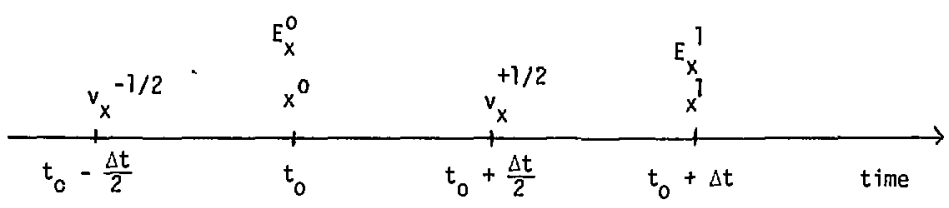

The upper indices are fractions of the basic time-step $\Delta t$. The positions and fields are defined at integral numbers of time steps, and the velocities (and currents) are defined at half time-steps; " $t_{0}$ " is the starting point of the simulation. The system is assumed to be periodic in $x$ for particles and fields and no loss of particles is allowed.

A basic one time-step loop in the simulation process works as follows:

(1) Find electric field from charge aistribution:

(a) Find Fourier components of $\rho(x)(\rho(x)$ is the charge density).

(b) Find field in Fourier space by using: $E_{x}=\sum Z_{k} \rho_{k} e^{j k x}$, where $E_{x}$ is the electric field, and $Z_{k}$ is a weight ing function of $k$.

(2) Push particles to new locations and find new charge distribution.

An external focusing force is applied in the bunched beam case. In the simplest case, it is proportional to the distance from the center of the bunch, when the space-charge force is linear. In this case, the strength constant of the focusing force is automatically determined by the code such as to balance the emittance and space-charge defocusing effects. The focusing farce is turned off when studying the coasting beam case. A resistive force can also be introduced. As a special case, it may be taken to be proportional to the linear charge density $o(x)$.

In the following section we describe in more detail the structure of the simulation code.

\section{(b) Technical Details af the Simulation Code}

The flow-chart of the code is presented in Figure 1. The system nas NX real cells and two dummy cells at both ends, to adjust boundary conditions. Initially, the electric field and charge density arrays are zeroed, to make place for the new values.

For the bunched bearn case, the length of the bunch is defined as: 


$$
L_{B}=2 \text {. DELX, }
$$

where DELX is in cell units (a cell $\Delta X$ is one unit length in the code).

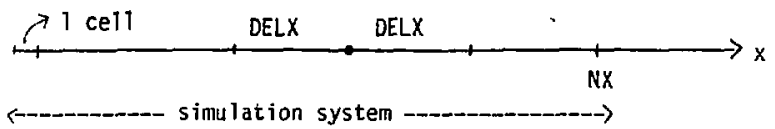

Then, a "phase-velocity" $\omega_{p}$ is defined by:

$$
\omega_{p}^{2}=4 \pi N^{T O T} q^{2} /\left(m . L_{B}\right)\left(\omega_{p}^{2} \equiv 4 \pi v_{p}^{2} ; v_{p} \text { is defined in Section III }\right) \text {, }
$$

where $\mathrm{N}^{\text {TOT }}$ is the total number of simulation particles, $q$ is the charge of one particle and $m$ is tie mass of one particle (taken to be equal to one). As a special case, when we investigate a parabolic density and velocity distribution ${ }^{(4)}$, the requirement of a stationary distribution function implies a linear focusing force:

$$
F=-K\left(x-x_{\text {initial center of bunch }}\right) \text {, }
$$

where $k$ is given by:

$$
K \equiv v_{0}^{2}=v^{2}+w_{0}^{2}
$$

Here:

$$
v=\frac{v_{x}, t h}{0 E L X}\left(v_{x}, \text { th is the maximum } x\right. \text {-velocity), }
$$

where $v$ is the emittance defocusing constant, and

$$
\omega_{0}=\sqrt{\frac{1.5}{2 \pi}} \cdot \frac{\omega_{p}}{D E L X},
$$

where $\omega_{0}$ is the space-charge defocusing constant. Both defocusing forces are linear.

The initial distribution function is given by ${ }^{(4)}$ :

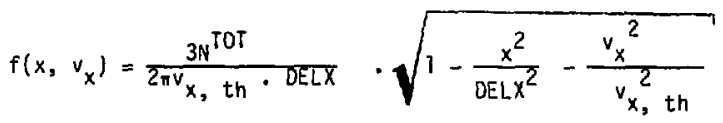


For the bunched beam case, initial particle positions and velocities are specified according to (1), which gives a parabolic distribution for both density distribution $p(x)$ and velocity distribution $f\left(v_{x}\right)$.

During the first time-step, the velocities are moved backwards half a time-step, for leap-frog centering purpose.

A variety of spatial and velocity distributions, such as gaussian or uniformly random distribution can also be provided by the code.

The initial charge distribution is found by proportional area-weighting of all particle contributions at the cell centers. The width of a particle is equal to the cell width. After initialization, periodicity is applied to the ends of the system:
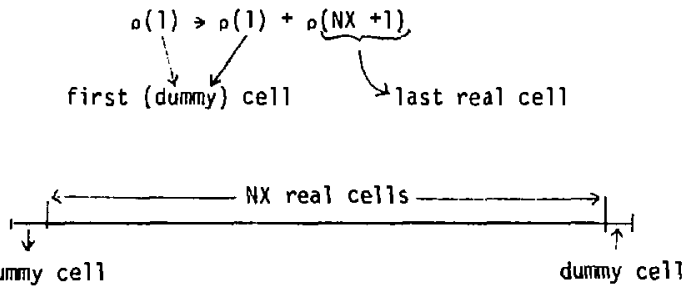

Now, the main time-integrating loop is started. Firsi, one calculates the riew field by the following steps:

(a) The average charge density is set equal to zera:

$$
\rho(x)=\rho^{o l d}(x)-\left\langle p^{o l d}(x)\right\rangle_{x}
$$

Therefore, $\rho(x)$ may have both positive and negative values.

(b) Calculate the Fourier transform of the charge density, using a sin-cas expansion:

$$
\begin{aligned}
& \rho(x)=\sum_{n} b_{n} \cos k_{n} x+\sum_{n} c_{n} \sin k_{n} x, \text { where: } \\
& k_{n}=\frac{2 \pi n}{N X},(n=1,2, \ldots)
\end{aligned}
$$


(c) Convert $p(x)$ to a complex exponential form:

$$
\begin{aligned}
& \rho(x)=\sum_{n} p_{n} e^{i k_{n} x}+\sum_{n} o_{n}{ }^{*} e^{-i k_{n} x} \text {, where } \\
& o_{n}=\frac{1}{N X} \int_{-\frac{N X}{2}}^{+\frac{N X}{2}} \rho(x) \exp \left(-i k_{n} x\right) d x
\end{aligned}
$$

and one obtains the relationships:

$$
\begin{aligned}
& b_{n}=o_{n}+o_{n}{ }^{\star}=\frac{2}{N X} \int_{\frac{N X}{2}}^{+\frac{N X}{2}} \rho(x) \cos \left(k_{n} x\right) d x \\
& c_{n}=i\left(\rho_{n}-\rho_{n}{ }^{\star}\right)=\frac{2}{N X} \int_{\frac{N X}{2}}^{+\frac{N X}{2}} \rho(x) \sin \left(k_{n} x\right) d x
\end{aligned}
$$

Therefore :

$$
\rho_{n}=\frac{1}{2}\left(b_{n}+\frac{c_{n}}{i}\right),
$$

and $p_{n}$ is usually complex:

$$
\rho_{n}=\rho_{n}^{\text {real }}+i_{n} \text { imag. }
$$

(d) Finally, one calculates the electric field from the usual longwavelength formula:

$$
E_{x}=-i \sum_{k} z_{k} o_{k} e^{i k x}
$$

The average electric field is set to zero. Then, we introduce gaussian shapes for the simulation particles:

$$
\rho^{1}(x) \text { of one particle } \sim \frac{e^{-x^{2} / 2 a_{x}{ }^{2}}}{\sqrt{2 \pi} a_{x}}\left\{0<a_{x}<1\right\rangle
$$

Here $x$ is measured from the center of each particle: $-1 / 2<x<1 / 2$. We take $a_{x}=1$ ("fat" particles). Also $\int_{-\frac{1}{2}}^{+\frac{1}{2}}{ }^{l}(x) d x=q$. In Fourier space, this 
corresponds to the multiplication of any Fourier component of the density o by a "smoothing" factor of $e^{-k_{n} a_{x}}$, which cuts off the highest $k$ 's.

From $(2)$, we have in Fourier space, including the smoothing factor:

$$
E_{k}=-i z_{k} a_{k} e^{-k^{2}} \quad\left(a_{x}=1, k_{n} \equiv k, n \rightarrow k\right)
$$

A resistive force can be introduced of the form:

$$
F^{R e S}=-q R p(x)
$$

For example, if the space-charge force is of the form:

$$
F^{\text {sp. ch. } ~}-g \frac{\partial p}{\partial x} \text {, then with resistivity, (and } g=1 \text { ), the electric }
$$

field in its Fourier form becomes:

$$
E_{k}=\left(i k p_{k}+R o_{k}\right)(-) e^{-k^{2}}
$$

Therefore:

$$
\begin{aligned}
& E_{k}^{\text {imag. }}=\left(k \cdot o_{k}^{\text {rea } 1}+R_{p_{k}}^{\text {imag. }}\right)(-) e^{-k^{2}} \\
& E_{k}^{\text {real }}=\left(-k \cdot \rho_{k}^{\text {imag. }}+R_{\rho_{k}}^{\text {real }}\right)(-) e^{-k^{2}}
\end{aligned}
$$

(e) The electric field is converted to a sin-cos form in fourier space, necessary for Fourier synthesis.

(f) The reverse fourier transform is performed, giving the electric field in real space, $E_{x}(x)$.

Boundary conditions for the field are specified as follows:

$$
E_{x}(N X+1)=E_{x}(1)
$$

External fields may be added at this point:

$$
E_{x} \rightarrow E_{x}+E_{x}^{e x t} \text {. }
$$

Finally, the source array is zeroed:

$$
p(x)=0
$$




\section{Particle Pushing}

If this is the first time-step, velocities are moved backwards by half a time-step, for time-centering purposes:

$$
v_{x}{ }^{-1 / 2}=v_{x}^{0}-\frac{\Delta t}{2} \cdot q E_{x}{ }^{w / m} \quad(m=1 \text { in the code })
$$

$E_{x}^{W}$ is the area-weighted field at the location of each particle.

For the bunched beam case, when a linear focusing force is applied, one obtains:

$$
\begin{aligned}
& v_{x} \rightarrow v_{x}-\frac{\Delta t}{m} k\left(x-x_{\text {initial }}^{\text {center of bunch }}\right) \\
& k \equiv v_{0}^{2}=v^{2}+\omega_{0}^{2} \text { (defined previous } i y \text { ) } \\
& m=1 \text {, in our code. }
\end{aligned}
$$

At this point, various diagnostics can be perfarmed on the particles, as follaws:

- Kinetic, thermal and drift energies are calculated and printed.

- Phase-space graphs are printed on-line.

- Velocity and density distributions are calculated.

Next, the actual particle pushing is done in a few steps:

(a) Area-we:ghted fields $E_{x}{ }^{W}$ are calculated at each particle position (center of particle).

(b) The velocities are advanced:

$$
\begin{aligned}
& \Delta v_{x}=\frac{q \Delta t}{m} \cdot E_{x}{ }^{W} \\
& v_{x} \rightarrow v_{x}+\Delta v_{x}
\end{aligned}
$$

(c) The positions are advanced:

$$
x \rightarrow x+v_{x} \cdot \Delta t
$$

(d) Check new positions:

Particles exiting the simulation system are pushed back, as follawing:

$x \rightarrow x-N X$, for particles exiting to the right.

$x \rightarrow X+N X$, far particles exiting ta the left.

Therefore, in principle loss of particles shauld never happen, unless particles are pushed too far to the right or to the left. 
The new charge distributioil $\rho(x)$ is calculated at cell center by area-weighting of particles in their new positions. The boundary conditions are established:

$$
\rho(1) \rightarrow \rho(1)+\rho(N X+1)
$$

Finally, the field diagnostics are performed, calculating and printing the Fourier components and the field energy. The time-integration loop is repeated for as many time steps as needed.

Because of the peculiarity of this $1-d$ code, which uses $E_{x}=\sum Z_{k} \rho_{k} e^{i k x}$ for the space-charge electric field, instead of the usual Poisson equation: $d E / d x=p(x)$, the electric field energy is also modified, as follows:

$$
\begin{aligned}
\text { Field Energy } & =\frac{1}{2} \sum_{k}{ }^{o_{k}}{ }^{*} \phi_{k} \\
& =\frac{1}{2} \sum_{k}{ }^{o_{k}}{ }^{\star} \frac{E_{k}}{i k}
\end{aligned}
$$

where $E_{k}$ is the electric field component, and $\rho_{k}$ is the compiex coefficient in the expansion:

$$
o(x)=\sum_{k}{ }^{o} e e^{i k x}
$$

Let:

$$
E_{k}=Z_{k} o_{k}
$$

Then:

$$
\begin{aligned}
\text { Field Energy } & =\frac{1}{2} \sum_{k} \frac{\rho_{k}{ }^{*} Z_{k}{ }^{\rho_{k}}}{i k} \\
& =\frac{1}{2} \sum_{k}\left|\rho_{k}\right|^{2} \frac{z_{k}}{i k}
\end{aligned}
$$

The reality of $E_{X}(x)$ implies:

$$
z_{k}^{*}=z_{-k}
$$


Therefore:

Field Energy $=\frac{1}{2} \sum_{i k>0}\left|p_{k}\right|^{2}\left[\frac{z_{k}}{i k}-\frac{z_{-k}}{i k}\right]$

Finally

Field Energy $=\sum_{k>0}\left|o_{k}\right|^{2}$ Im $\frac{z_{k}}{k}$

In particular, if $\operatorname{Im} Z_{k}=k$, then:

Field Energy $=\sum_{k>0}\left|\rho_{i}\right|^{2}$

The resistive farce does work on the system at a rate of:

$$
\frac{d E^{R e s}}{d t}=2 \operatorname{Re} \sum_{k>0} o_{k}^{*}\left(\frac{R}{i k}\right) \dot{o}_{k}
$$

The total energy conservation is. expressed as:

Kinetic Energy + Field Energy $+\frac{1}{2} \int_{0}^{x} v_{\text {ext }}{ }^{d d x}+\int_{0}^{t} d t \frac{d \varepsilon^{R e s}}{d t}=$ constant (at time $t$ )

\section{Conversion from Code Units to Physical Units}

As an example, let us consider a case with a space-charge force of the form $-g \frac{\partial \rho}{\partial x}$. Let us define a phase velocity $v_{p}$ in code units:

$$
v_{p} \equiv \sqrt{\frac{n q^{2}}{m}} \quad \text { (all quantities in code units) }
$$

(The relation between this $v_{p}$ and $\omega_{p}$ defined in Section $I I-(b)$ is: $\omega_{p}{ }^{2}=4 \pi v_{p}{ }^{2}$ ) $n$ is the number of simulation particles per cell, $q$ is the charge per particle and $m$ is 1 . ( $\rho \equiv q n$ )

Then, the equation of motion used by the computer is (a)l quantities are expressed in computer units):

$$
\text { m } \frac{d x^{2}}{d t^{2}}=-q^{2}\left(g \frac{d n}{d x}+R \cdot n\right)(\text { code units; } g=1)
$$


$R=R^{\prime}$. $V_{B}$, where $R^{\prime}$ is the resistance per unit length, and $v_{B}$ is the bean velocity. This equation is also true in CGS units.

In MrS-C units, the phase velocity becomes:

$$
\vec{v}_{p}=\sqrt{\frac{\bar{n} \bar{q}^{2}}{m 4 \pi \varepsilon_{o}}},
$$

i

and the equation of motion becomes:

$$
\bar{m} \frac{d^{2} \overline{\bar{x}}}{d \bar{t}^{2}}=-\frac{\bar{q}^{2}}{4 \pi \varepsilon_{0}}\left(g \frac{d \bar{n}}{d \bar{x}}+4 \pi \varepsilon_{0} \bar{r} \cdot \bar{n}\right), \quad\left(\bar{R} \equiv R^{t} \cdot \bar{v}_{B}\right)
$$

Al: barred quantities are in MKS-C units.

Let: $\bar{x}=r_{L} \cdot x$

$$
E=r_{T} \cdot t
$$

Then: $\bar{v}_{p}=r_{L} \cdot r_{T}^{-1} v_{p}$

$$
\begin{aligned}
& \bar{R}=r_{L}^{-l} \cdot \frac{R}{4 \pi \varepsilon_{0}} \\
& \bar{k}=r_{L}^{-1} k
\end{aligned}
$$

Therefore, this is the simplest possible correspondence between code units and MKS-C units, which gives:

$$
\frac{\mathrm{R}}{\mathrm{kg}}=\frac{4 \pi \varepsilon_{0} \overline{\mathrm{R}}}{\mathrm{kg}}
$$

The ratio between resistive and space-charge forces is kept the same, both in code and MKS-units.

Therefore:

$$
\left.\begin{array}{rl}
\vec{v} & =r_{L} r_{T}^{-1} v \quad \text { (for any velocity) } \\
\text { and } \quad \vec{\omega}=r_{T}^{-1} \omega, \text { where } \omega=\omega_{k}+i_{\gamma_{k}}
\end{array}\right\}
$$

$\gamma_{k}$ is the growth rate and $\omega_{k}$ is the rea? irequency for mode $k$ (unbarred quantities are in code units). 
In order to interpret a simulation, one may first find $r_{L}$ from:

$$
\bar{k}=r_{L}^{-1} k
$$

For finite physical and compucer systems (of lengths $[$, $L$ respectively), we have:

$$
\bar{k}_{\min }=r_{L}^{-1} k_{\min } \text {, or } r_{L} \cdot \frac{k}{k}=\frac{\bar{L}}{L} \text {, }
$$

where: $k_{\min }=\frac{2 \pi}{L}, \bar{k}_{\min }=\frac{2 \pi}{I}$, and this $r_{L}$ value is valid for the entire k-spectrum.

Once $r_{L}$ is known, $R$ (to be used in the simulation) is found from:

$$
R=r_{L} \cdot 4 \pi \varepsilon_{0} \cdot \bar{R}
$$

Then:

$$
r_{T}=r_{L}\left(v_{p} / \bar{v}_{p}\right)
$$

where $v_{p}$ and $\bar{v}_{p}$ are given quantities. Therefore $\bar{v}, \bar{w}_{k}$ or $\bar{y}_{k}$ can be found, once the corresponding simulation values are known, by using ( $A$ ).

One can change the parameter $q$ in the simulation, in order to avoid problems caused by initial noise or nonlinear effects, for certain ranges of $R$. This will change $r_{T}$, if $r_{L}$ remains the same. If we want to change $r_{L}$ by requiring a certain value for $R$, then (for given $k$ ), the simulation length (number of simulation cells) will have to be changed accordingly, to preserve the ratio between the resistive and space-charge forces. However, the simulation length (and also the total number of particles in the system) cannot be changed too much (at most by an order of magnitude).

Also, $q$ or $\Delta t$ cannot be increased too much, if we require (to avoid numerical instabilities):

$$
\Delta v_{x} \leqslant v_{x}
$$

and

$$
v_{x} \Delta t \leqslant \Delta x \text {, where } \Delta x \text { is the length of a simulation cell. }
$$

Therefore, in the case where $q$ or $\Delta t$ or the simulation length cannot be changed too much, it might become necessary to perform lengthy simulations for 
weak instabilities, which usually require either a large number of particles or a sophisticated method to produce quiet starts with relatively modest number of simulation particles.

\section{References}

(1) Proceedings of the HIF Workshop, 0ct. 29 - Nov. 9, 1979, LBL-10301, Sept. 1980.

(2) L. Smith et a1., LBL Reports HIFAN 13, 14 and 15 (1977).

(3) L.J. Laslett, et a1., LBL-8351, November 1978.

(4a) A. Hofmann, CERN 77-13, p. 159 (Ju 1y 19, 1977).

(4b) D. Neuffer, Particle Accelerators, Vol. 11, 23-26 (1980).

(5) P. J. Channel1 et al., LBL-11367, Ju1y 1980.

\section{Acknowledgement}

This work was supported by the Director, Office of Energy Research, Otfice of Inertial Fusion, Research Division of the U.S. Department of Energy under Contract No. W-7405-ENG 48 . 
Fig. 1: Flow Chart of the Simulation Code

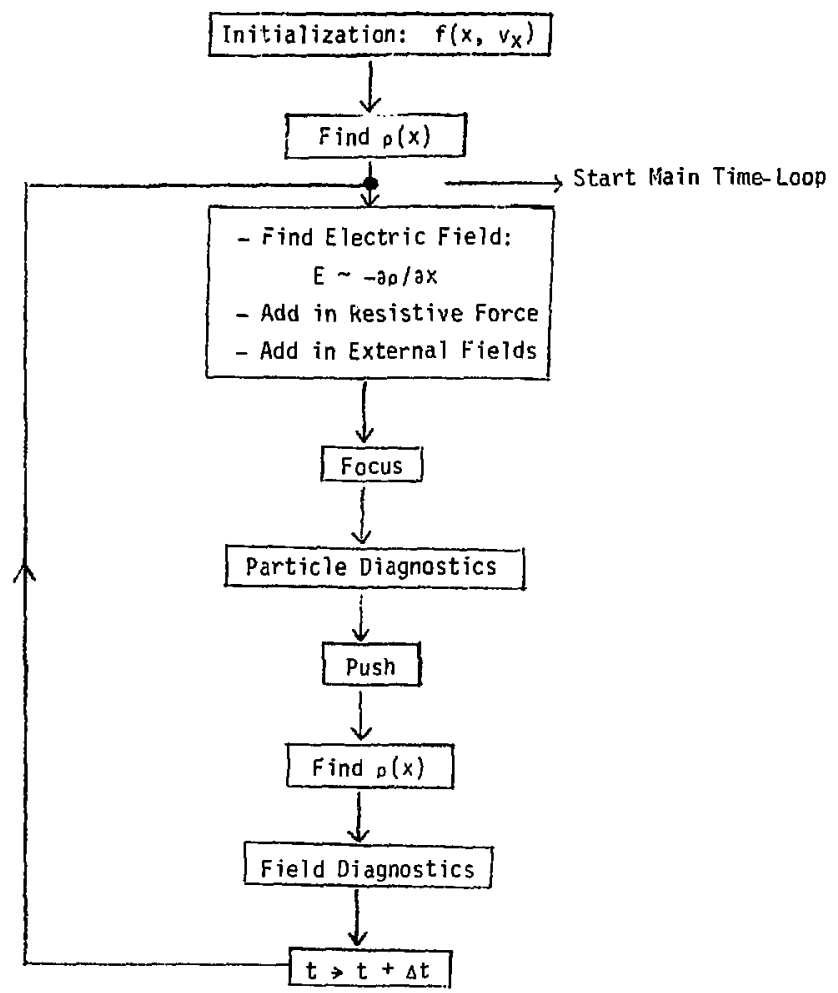

